Bioscientia Medicina: Journal of Biomedicine \& Translational Research

Journal Homepage: www.bioscmed.com

\title{
Acute Colonic Pseudo-obstruction (Adynamic Ileus) after Cesarean Section:
}

\section{A Case Series}

\author{
Sulistiari Retnowati ${ }^{*}$, Ivanna Beru Brahmana²
}

${ }^{1}$ Department of Obstetrics and Gynecology, PKU Muhammadiyah Gamping Hospital, Sleman, Indonesia

2 Department of Obstetrics and Gynecology Faculty of Medicine and Health Sciences, Muhammadiyah Yogyakarta University, Bantul, Indonesia

\section{A R T I C L E I N F O}

\section{Keywords:}

Acute colonic pseudoobstructive

Distended abdomen

Adynamic ileus

Cesarean section

Case series

\section{*Corresponding author:}

Sulistiari Retnowati

\section{E-mail address: \\ sulistiariretnowati@gmail.com}

All authors have reviewed and approved the final version of the manuscript.

\begin{abstract}
A B S T R A C T
Background. Maternal mortality is still high. The case that we will report below is an example of cases that can be happened in the hospital and can be ended with maternal mortality. Case presentation: A 30 years old disabled primigravida with ileus post- Caesarean Section day 5 was managed by relaparotomy and partial resection of caecum and ileum due to intestinal perforation, and die on the fifth days after relaparotomy due to sepsis. A-33 years old secundigravida with history of relaparotomy on 4 days after caesarean delivery in the first pregnancy due to ileus, was performed re-caesarean section due to unprogressed labor. Twelve hours after the operation the abdomen becomes distended and can be managed successfully by conservative early diagnosis management. A 29 years old primigravida has performed caesarean section with history of myomectomy 3 years ago. Twenty four hours after Caesarean Section, the abdomen becomes distended and early conservative management was done, but at 72 hours after the operation, there was intestinal perforation and performed colostomy. Conclusion: Acute colonic pseudoobstructive after caesarean section is a rare case, and delayed diagnosis and management increase the morbidity and mortality.
\end{abstract}

https://doi.org/10.37275/bsm.v6i4.489

\section{Introduction}

Acute colonic pseudoobstruction (ACPO) or Ogilvie Syndrome was first described by Sir Haneage Ogilvie in 1984, It has symptoms and signs of large bowel obstruction in the absence of obvious mechanical obstruction. Delay in diagnosis can lead to caecal perforation which is associated with significant morbidity and mortality ${ }^{1,2}$. The clinical condition that is easily seen in ACPO is the presence of abdominal distension, which in this case occurred in a patient after a Caesarean section operation ${ }^{1}$.

ACPO reportedly occurs in up to 1 in 1500 deliveries, but the true incidence following cesarean section is unknown. In 2000-2002 three maternal death from the condition were reported in the Confidential Inquiries into Maternal death reports. ${ }^{1,2}$

There were 3 cases of abdominal distension following cesarean section described here, in which one case ended in maternal death due to ileo-caecal perforation, one case improved with conservative therapy, and one patient underwent a colostomy by the digestive surgery department. 


\section{Case Presentation}

\section{Case 1}

A 30 years old primigravida was referred from other hospital with suspected ileus after the fifth day of cesarean section on indication cephalopelvic disproportion. The patient is a disabled person, with a scoliosis disorder, and deformed lower limbs. There is a history of constipation during pregnancy. After cesarean section, she received antibiotic injection, ketorolac, alinamine and ranitidine.

On physical examination, the general condition was weak, the abdomen was very distended, blood pressure $115 / 80 \mathrm{mmHg}$, heart rate 105 beats/minute, and temperature $38^{\circ} \mathrm{C}$. Normal heart and lung examination, abdominal wall higher than chest wall, weak peristaltic, good surgical wound suture, and normal puerperal vaginal disccharge. On laboratory examination, the results obtained haemoglobin 10, $2 \mathrm{~g} / \mathrm{dL}$, white blood cell count 13,600 / mmk, hypokalemic with $\mathrm{K} 2.5 \mathrm{mmol}$ / L (N 3.5-5.1). Abdominal X-ray examination showed a pneumoperitoneum image.

Correction of potassium was performed because of hypokalemia, and continued with laparotomy due to suspicion of bowel perforation. During the operation, perforation was found in the caecum and ileum, then a partial wedge resection was performed, continued with anastomose. The tissue was sent to the laboratory. The patient was admitted for two days in the Intensive Care Unit (ICU), died on the fifth postoperative day due to sepsis.

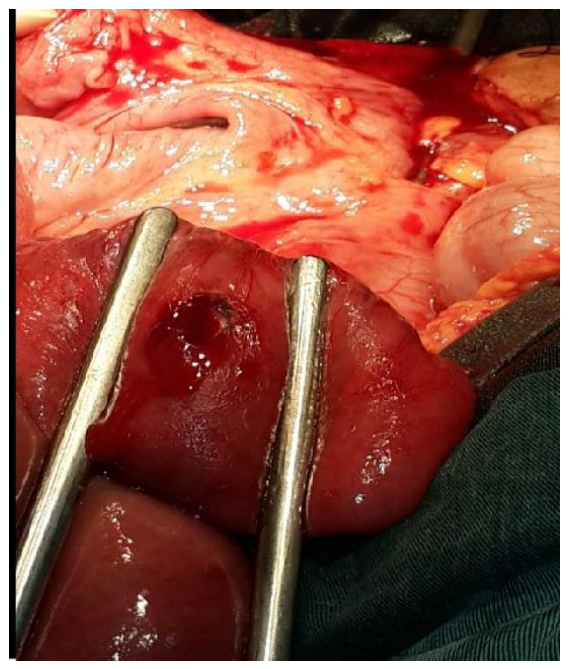

Figure 1. Ileum perforation after cesarean section

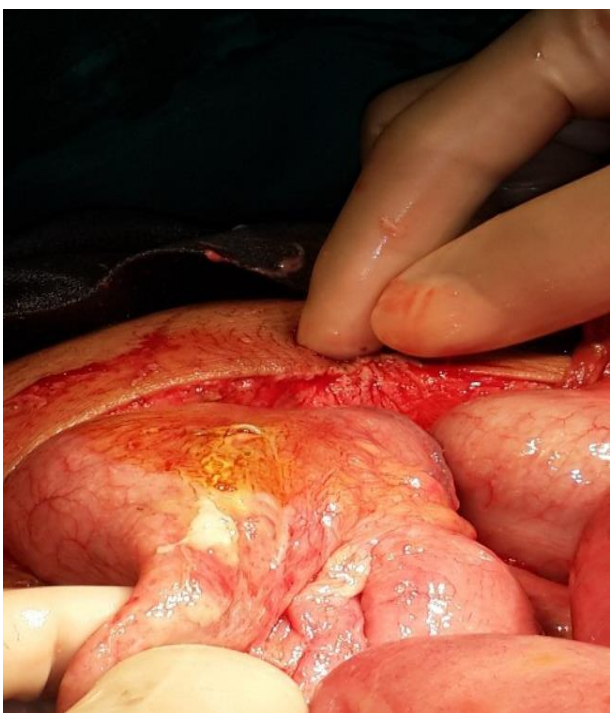

Figure 2. Caecal perforation after cesarean section 


\section{Case 2}

A 39 -week pregnant, 33 year old, second-gravida patient, came to the Emergency room with the examination showed the cervical dilatation was $3 \mathrm{~cm}$, the head is still floating. The patient had a history of cesarean section 3 years ago. The first cesarean section, performed at Hospital A, then on the fourth day after surgery an ileus occurred and was referred to Hospital B, and performed relaparotomy by the Surgery department. The patient wanted to be able to deliver vaginally because the patient scare with cesarean section

Re-cesarean section operation was carried out based on the indication of unprogress labor. The operation went smoothly, under spinal anesthesia, and the drugs used for the operation were decain, ketorolac, pethidine, and ondansetron. Post-surgery, the patient given antibiotic therapy, tramadol injection, and injection prostigmine $0.25 \mathrm{mg}$ intramuscularly two times a day. Twelve hours after the operation, the patient reported complaining of bloating, not flatus, and the stomach was distended after eating and drinking. Physical examination revealed increased peristalsis, absent metallic sound, tenderness throughout the abdomen, and epigastric tenderness.

The results of observations showed that there was no improvement in patient complaints, there was still bloating. What happened at that time is a similar events with the history of previous operations, the patient was subjected to abdominal X-rays and electrolyte examination. The patient received prostigmine injection therapy $0.5 \mathrm{mg}$ intravenously injected slowly. Stop eating and drinking, put in a naso gastric tube, metochlorpropamid injection, and change analgetic tramadol with ketorolac.

Electrolyte test results are within normal limits. Complaints of bloating immediately improved after receiving prostigmine injection. On the second postoperative day, flatus was reported frequently, and the patient was allowed to sit mobilization. On the third day, Naso Gastric Tube was removed, and allowed to go home on the fourth day. Patient oral therapy was erythromycin $500 \mathrm{mg}$ t.d.d and metochlorpropamide t.d.d.

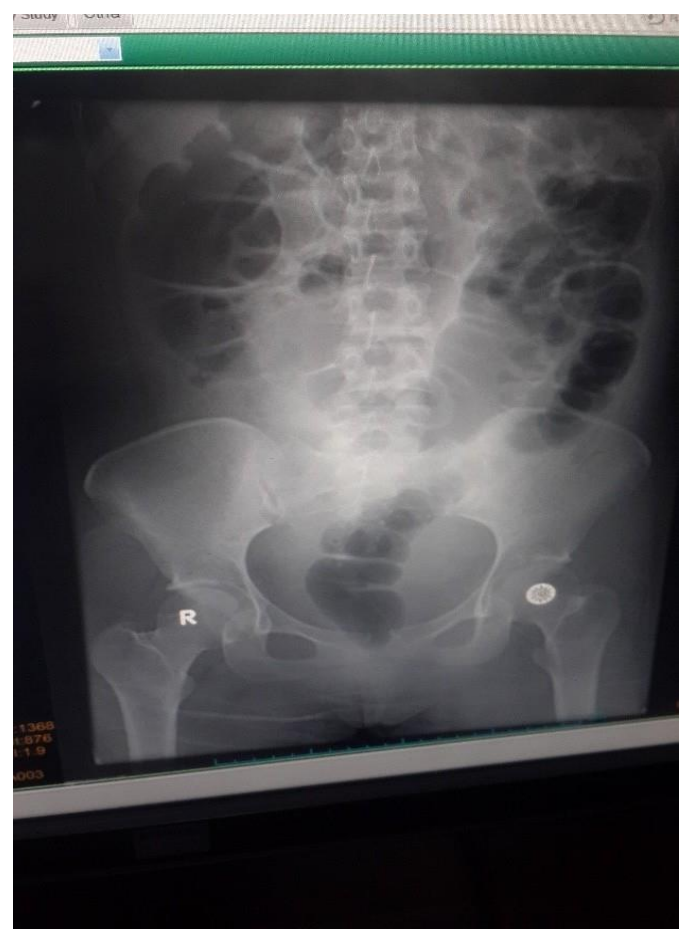

Figure 3. Antero posterior supine abdomen $\mathrm{X}$ ray position in a patient with bloating 12 hours after surgery 


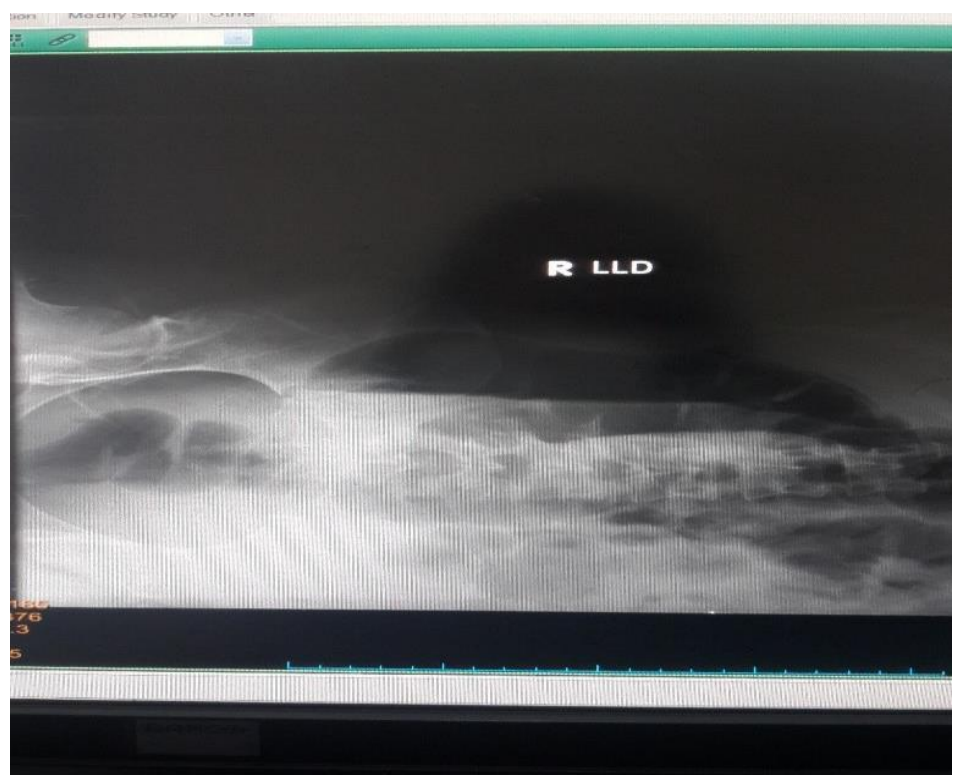

Figure 4. Left lateral abdomen X-ray position in a patient with bloating 12 hours after surgery

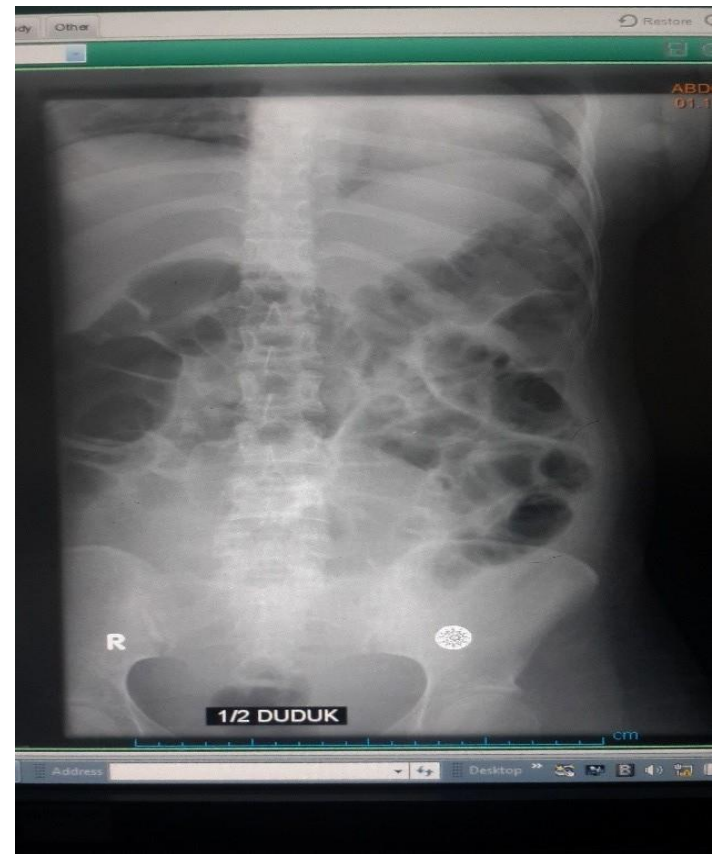

Figure 5. Postero anterior erect X-ray in a patient with bloating 12 hours after surgery.

\section{Case 3}

A primigravida aged 29 years, 38 weeks of gestation, performed elective cesarean section and Intra Uterine Device insertion by indication pregnancy with history of myomectomy. Laboratory results showed Haemoglobin levels of $11 \mathrm{~g} / \mathrm{dL}$ and leucocyt $14,6 \times 10$ 9/L. The operation went smoothly and received ketorolac analgesic therapy, 24 hours postoperatively, the patient complained of bloating, and was given intramuscular $0.25 \mathrm{mg}$ of prostigmine therapy, metochlorpropamide injection. On the second day post surgery, bloating did not improve, the peristalsis were weak, patients complains vomiting, and fever. The patient was fasted and Naso Gastric Tube was placed for decompression.

On the third day post operation, blood laboratory tests obtained Haemoglobine $10.3 \mathrm{~g} / \mathrm{dL}$, leukocytosis 30,000 / mmk), normal electrolytes. The patient was consulted to a Digestive Surgery departement, with a 3-position abdominal X-ray obtained a 
pneumoperitoneum Relaparotomy and colostomy was performed. The patient's condition improved after colostomy.

\section{Discussion}

Acute colonic pseudo obstructive (ACPO) or adynamic ileus is a colonic motility disorder. The pathophysiology of postoperative ACPO is not known certainty, involving the balance of the sympathetic and parasympathetic nerves. Under normal conditions the parasympathetic nerves increase the contractility of the colon, while the sympathetic nerves decrease motility. Lack of parasympathetic activity underlies the incident, based on an improvement in ACPO conditions on parasympathomimetic (neostigmine) administration. $1,2,3$

The presence of neuropraxia of the parasympathetic nerve fibers at Sacral 2-4 (S2-4) is believed to underlie the pathology of this complaint. It passes through the inferior hypogastric plexus, which is located close to the cervix, vagina, and broad ligament, before innervating the left colon. The plexus can be injured during surgery, childbirth, or trauma. The presence of atony distal to the colon gives the appearance of mechanical obstruction. Peristalsis in the proximal colon is inhibited by dilatation of the distal colon. The pathogenesis of events in these cases is multifactorial. ${ }^{1,2}$

Pregnancy increases the risk of ACPO. Gravid uterus can compress the parasympathetic sacral plexus, progesterone decreases intestinal motility, in addition to increased sympathetic nerve activity. The delivery process also increases the risk by increasing prostaglandins and cytokines. Caesarean section with ischemia, inflammation, or parasympathetic sacral damage is a major contributor to ACPO. Emergency cesarean sections increase the incidence of ACPO due to ischemia due to bleeding, and inflammatory reactions from a high stress response. 1,4

The initial signs of ACPO are: abdominal pain, bloating with abdominal distension, with increased or decreased bowel movements. Symptoms appeared within 48 hours postoperatively, and most rapidly appeared within 6 hours postoperatively. Mild fever, tachycardia, increased or decreased bowel sounds, with increased bloating or abdominal distension are later complaints. Symptoms may be accompanied by nausea, vomiting, diarrhea or constipation. On further examination, there is an increase in CRP, leukocytosis, and electrolyte disturbances (hypokalemia, hypomagnesia, and hypocalcemia). 1,5,6,7

Clinical signs that support the ACPO diagnosis are very pronounced abdominal dilation with minimal nasogastric aspiration, accompanied by persistent bowel movements in the form of diarrhea or hearing bowel sounds. Symptoms in the intestine vary widely from normal bowel sounds, hyperactivity or disappearance, some patients can still be flatus. $3,8,9$ The sign that differentiates ACPO from ileus is difficult, but ileus tends to present with less severe bowel distension, and is more often associated with loss of bowel sounds and absence of flatus. ${ }^{1,8}$

A useful initial investigation is X-ray examination of the abdomen and thoracic, which shows dilatation of the colon and caecum. The ileus generally affects the small intestine, so that small bowel distension is more supportive of the ileus image. Air fluid level images are generally associated with mechanical obstruction or ileus, but were found in $42 \%$ of patients with ACPO. Contrast administration can distinguish the presence of mechanical obstruction or not. Contrast abdominal and pelvic CT scans provide a more detailed picture, and a more accurate measurement of the caecum diameter. Examination of chest and abdominal x-rays with a pneumoperitoneum image gives a picture of perforation. ${ }^{1,10,11}$

Cho et al reported 4 cases of adynamic ileus after CS associated with: (1). there is massive bleeding that causes hemodynamic disturbances, resulting in disruption of blood circulation in the intestines and colon, due to the priority of blood flow to vital organs; (2). severe constipation, which causes fecal buildup, intestinal dilation, and exacerbation of slow bowel movements; 12 (3) use of meperidine as a postoperative analgesic;13.(4) CS with intestinal manipulation, adhesiolisis, tubal ligation, hysterectomy and severe vomiting during surgery as a result of unstable 
hemodynamic status. Of the 925 CS operations without bleeding in 6 years there were no cases of postoperative adynamic ileus, whereas 3 of 71 post-CS patients with bleeding more than $2000 \mathrm{~mL}$ had ileus. ${ }^{12}$

In the first case there was a history of constipation in the patient, while in the second case the risk factor was the use of opioid drugs, in the form of pethidine during surgery, and tramadol as a postoperative analgesic. Risk factors such as bleeding or excessive manipulation of the intestine were not identified. It cannot yet be explained in detail the cause of the recurrence of cases as happened in the second patient. In all three cases there was no bleeding during the operation. The second and third patients had a history of previous operations. The three patients were young, there were no other comorbid diseases except for the disabled patient in the first case. The first and third patients were an elective CS procedure, while the second patient was an emergency CS procedure.

Cho et al mentioned that pregnant women with chronic constipation, administering constipation therapy during pregnancy, and administering enemas before CS reduce the risk of post-SC ileus ${ }^{12}$. Based on previous publications, postoperative ileus is treated with supportive management including: fasting, correction of fluid and electrolyte disturbances, early mobilization of the patient, NGT insertion, enema administration, laxatives, discontinuation of drugs that interfere with colonic motility such as opioid analgesics anticholinergic and calcium antagonists, and use neostigmine (prostigmine).1,2,12- 17

Some agents are said to work as prokinetic agents to treat postoperative ileus, Cisapride is a serotonin agonist that facilitates the release of acetylcholine, however its use in some countries has been withdrawn because of its cardiac side effects Ceruletide is a synthetic peptide that increases bowel movements by acting as a cholecistokinin antagonist. Metochlorpropamide is said to be effective, although not all authors agree. 16,18,19 Metylnatrexone can block the negative effects of opioids on the intestine without reducing the analgesic effect, and is used as a therapy in cases of constipation induced by opioids. Alvimopan, an opioid receptor antagonist, is used to enhance recovery of the upper and lower gastrointestinal tracts after bowel resection. Metylnatrexone, a derivative of naltrexone and alvimopan is a new drug drug that can be an important component in the perioperative management of postoperative ileus. ${ }^{16,18}$ Use of NSAID analgesics improves postoperative ileus because of its anti-inflammatory effect on reducing nausea and vomiting and improving gastrointestinal transit. ${ }^{18}$

Cochrane review of 39 studies states that most of the prokinetic agents used in the management of bowel function return after major abdominal procedures are not supported by some evidence. The new drug Alvimopan, shortens the period of return to bowel function, however many studies have failed to report on the current methodology based on the current protocol. Erythromycin, cholecystokinin, cisapride, dopamine antagonists, propranolol are not sufficiently shown to have an effect, intravenous administration of lidocaine, and neostigmine appear to be of some benefit, but need further verification. 19

Early postoperative feeding stimulates reflexes that produce coordinated propulsive activity and induces gastrointestinal hormone secretion which has a positive effect on bowel movements, Cho et al said chewing gum can also be used in supportive management. ${ }^{12,18,20}$ Fake food from chewing candy The gum causes vagal cholinergic stimulation of the gastrointestinal tract and induces salivary release of gastrin, pancreatic polypeptides, and neurotensin, all of which increase gastrointestinal movement.18,20

Epidural anesthesia has a beneficial effect because it blocks the stimulation of afferents that trigger an endocrine metabolic stress response to surgery, thereby inhibiting the catabolic activity of the hormones released. In addition, epidurals increase blood flow to the intestines and have anti-inflammatory effects. In a thoracic epidural study with bupicavaine, there was a significantly reduced incidence of postoperative ileus when compared to postoperative opioids. ${ }^{18}$

Pharmacological therapy that has positive effects and has been shown to be beneficial in RCT studies is the administration of neostigmine ${ }^{1,2,21}$. Neostigmine is 
a reversible acetylcholinesterase inhibitor, which directly stimulates muscarinic receptors, thereby increasing bowel movement activity ${ }^{13}$. Neostigmine improves autonomic nerve balance, by inhibiting acetylcholine activity to improve transmission of impulses through the myoneural junction in the intestinal wall. ${ }^{2}$

Side effects of prostigmine include: vomiting, bronchoconstriction, bradycardia, hypotension and syncope. Atropine sulfur should be available as an antidote to muscarinic effects. Neostigmine is injected intravenously within 3-5 minutes, the patient is in a sleeping position, and under the supervision of a doctor. Vital sign measurements are taken within 1530 minutes after the drug is administered. Contraindications to neostigmine administration are intestinal obstruction, bowel perforation or ischemia, pregnancy, uncontrolled cardiac arrhythmias, bronchospasm, and renal impairment.12,13

Intravenous neostigmine has a rapid onset of 1-20 minutes, and a short duration of 1-2 hours. Clinical improvement was seen within 3 hours in the form of decreased abdominal distension and radiological reduction in colon diameter. If there is recurrence, the administration can be repeated one more time. Patients who fail to respond to the second dose require surgical decompression of the colon. ${ }^{13}$

Supportive therapy has been carried out in the three patients above, the patient was fasted after starting to develop bloating and was put in NGT, the use of opioid drugs, in the form of tramadol, in the second patient was stopped and replaced by nsaid. In the second and third patient antibiotics therapy and metochlorpropamide were also administered. However, supportive therapy alone did not produce results. All three patients underwent CS under spinal anesthesia, none underwent epidural anesthesia.

In the first patient there was no history of neostigmine administration, the second and third patients were given neostigmine. In the second case the administration of neostigmine improved the patient's condition so that the bloating was reduced rapidly, in the third patient the patient was given neostigmine, but intestinal perforation occurred.

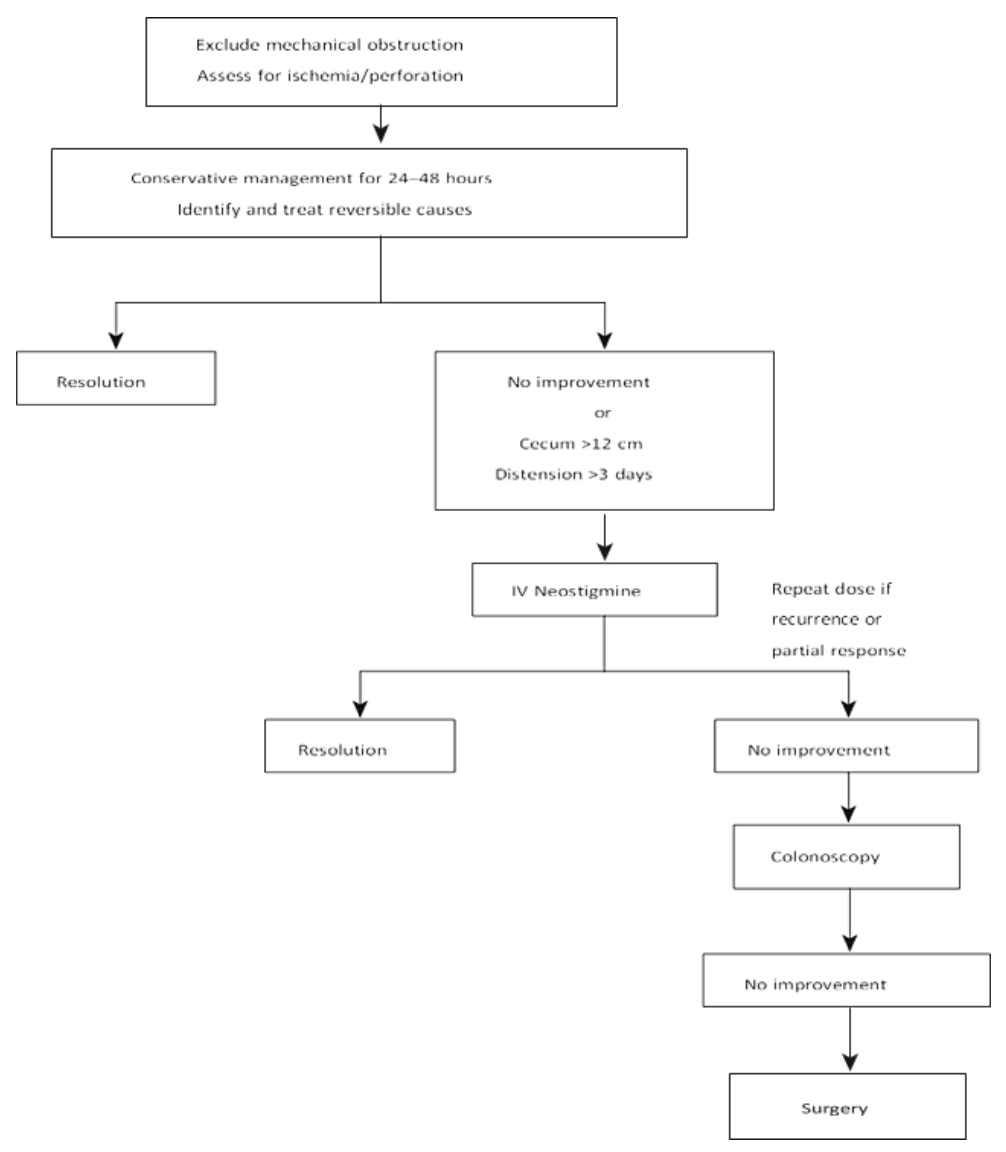

Figure 6 Algorithm for the management of $\mathrm{ACPO}^{7}$ 
An evidence-based guideline for the treatment of ACPO was recently published by the American Society for Gastrointestinal Endoscopy. ${ }^{7}$ The guidelines recommend conservative therapy as the initial preferred management, based on observational studies only. Potentially contributory metabolic, infectious and pharmacologic factors should be identified and corrected. Active intervention is indicated for patients at risk of perforation and/or failing conservative therapy. Neostigmine is effective for the majority of patients Colonic decompression is the initial invasive procedure of choice for patients who fail neostigmine therapy or for whom this drug is contraindicated. Surgical decompression should be reserved for patients with peritonitis or perforation and for those who fail endoscopic and medical therapy.A proposed algorithm for the management of ACPO is detailed in figure 6.

According to the diagram in Figure 6, conservative therapy should be administered within 24- 48 hours postoperatively before neostigmine is given, excluding any mechanical obstruction at the start of management. The third patient had a history of previous myomectomy surgery, so that the possibility of adhesions causing mechanical obstruction cannot be ruled out. The patient was subjected to relaparotomy by the digestive surgery department at another referral hospital so that the exact cause of bloating which ended in perforation in the third patient could not be ruled out. Identification and measurement of bowel diameter is very important examination, because caecum diameter of more than $10 \mathrm{~cm}$ for a long time (> 3-4 days), and in patients who do not improve after supportive therapy within 24-48 hours require surgical intervention. 22,23

In the first case conservative therapy is carried out for more than 48 hours. The patient was referred to our hospital on the fifth day of CS with the condition that bowel perforation had occurred. In all three patients, abdominal $\mathrm{x}$-rays had been performed, but the intestinal diameter had not been measured.

Measurement of bowel diameter can help determine the need for immediate surgical intervention. The caecum is the part of the colon with the largest diameter, so that if there is an increase in pressure its size will increase rapidly, compared to other parts of the colon. Caecum rupture usually occurs in the serous part between taenia, due to mechanical strain ${ }^{2}$. Several case reports mention the incidence of post-CS caecum perforation as reported by Nadarajah et al, Laskin et al Deshpande et al.8,9,24

In patients who do not improve with conservative decompression therapy, it is done by endoscopy (decompression colonoscopy). Operative action is indicated in patients with signs of intestinal ischemia or perforation. The type of surgery depends on the condition of the intestine, If the intestine condition without ischemia or perforation performed caecostomy, while if the condition was perforation performed resection. 13

The success rate for supportive management is around $85 \%$, while the mortality rate is $15 \%$ with early diagnosis and intervention, but the mortality rate increases to $36-50 \%$ when intestinal perforation and ischemia have occurred. ${ }^{13}$

In this case report, the first patient had bowel perforation and died on the 5th day of the relaparotomy due to sepsis, while the bowel perforation that occurred in the third patient improved after resection and colostomy. The second patient was managed successfully with conservative therapy

\section{Conclusion}

Postoperative acute colonic pseudoobstructive after Cesarean section is a rare case, if it is not diagnosed and handled properly, may increase maternal morbidity and mortality. The pathophysiology and risk factor for ACPO is unclear, so high suspicion is needed when found post cesarean patient with progressive abdominal distension

\section{References}

1. Hughes AE, Smart NJ, Daniels IR. Acute colonic pseudo-obstruction after caesarean section: a review and recommended 
management algorithm. Obstet Gynaecol. 2019; 21: 283-290.

2. Aparna, A., Helena, K., Asha, P., Mark, J., Alok, G. Review acute colonic pseudo- obstruction after caesarean section learning objectives. Obstet Gynaecol. 2006; 8: 207-13.

3. Platt JA, Rossi CM. Ogilvie syndrome after cesarean section. J Am Acad Physician Assist. 2015; 28: 1

4. Scott, L. Gastrointestinal disease in pregnancy in creasy. In Resnik: Maternal Fetal Medicines Priciples And Practise. WB Saunders Company. 1994.

5. Fatnassi R, Mkhinini I, Abdelkefi MS, Torki EHS. Ogilvie's syndrome: A caesarean section complication (a two cases report and review of the literature). EC Gynaecol. 2015; 1: 35-40.

6. Kotsev, S. Ogilvie's syndrome following cesarean delivery: The Dubai's case. Saudi J Anaesth. 2011; 5: 335-8.

7. Cunningham F. Williams Obstetric. 2005. New York. Lippincott.

8. Nadarajah. Ogilvie's syndrome with caecal perforation following caesarean section: A case report. J Med Cases. 2013; 4: 1-4.

9. Deshpande G, Hariprasad, B. , Narasamma, U. A Postoperative complication following laparotomy for ruptured uterus. 2012; 16: 1-5.

10. Henry K. Adynamic ileus. Radiopaedia Org 2020; 11.

11. Cho FN, Liu CB, Li JY, Chen SN, Yu KJ. Adynamic ileus and acute colonic pseudoobstruction occurring after cesarean section in patients with massive peripartum hemorrhage. J Chinese Med Assoc. 2009; 72: 657-62.

12. Saunders MD, Kimmey MB. Ogilvie's syndrome. 2004; 19: 303-10.

13. Kammen BF, Levine MS, Rubesin SE, Laufer I. Adynamic ileus after caesarean section mimicking intestinal obstruction: findings on abdominal radiographs. Br J Radiol. 2000; 73: 951-5.

14. Elsebay L, Galal MA. Ogilvie's syndrome after cesarean section: Case report In Saudi Arabia and management approach. Case Rep Obstet Gynecol. 2017; 1-5.

15. Erowele GI, Sise T. Treatment options for postoperative ileus. US Pharm. 2010; 35: 5573.

16. Chelmow D, Aronson MP. Intraoperative and postoperative complication of gynecologic surgery. In: Current Diagnosis and Treatment. 2007.

17. Luckey A, Livingston E., Tache Y. Mechanisms and treatment of postoperative ileus. Arch Surg. 2003; 138: 206-14.

18. Traut U. Systemic prokinetic pharmacologic treatment for postoperative adynamic ileus following abdominal surgery in adults. Cochrane Database Syst Rev. 2008 Doi:10.1002/14651858.CD004930.Pub3.

19. Ahmed MR., Sayed-Ahmed WA, Khamess RE, Youwakim MS, El-Nahas KM. Efficacy of three different regimens in recovery of bowel function following elective cesarean section: A randomized trial. J Perinat Med. 2018; 46: 78690.

20. Ponec R. Neostigmine for the treatment of acute colonic pseudo-obstruction. NEJM 1999; 341: 137-41.

21. Naveed M. American society for gastrointestinal endoscopy guideline on the role of endoscopy in the management of acute colonic pseudo-obstruction and colonic volvulus. Gastrointest Endosc. 2020; 91: 22835.

22. Norton-Old KJ, Yuen N., Umstad MP. An obstetric perspective on functional bowel obstruction after cesarean section: A case series. J Clin Gynecol Obstet. 2016; 5: 53-7.

23. Salamah AK. Ileo-caecal volvulus postcesarean section: A case report. Saudi J Gastroenterol. 2000; 6:163-4.

24. Laskin MD, Tessler K, Kives S. Cecal perforation due to paralytic ileus following primary caesarean section. J Obstet Gynaecol Canada. 2009; 31: 167-71. 